PROCEEDINGS OF THE

AMERICAN MATHEMATICAL SOCIETY

Volume 137, Number 5, May 2009, Pages 1777-1782

S 0002-9939(08)09715-3

Article electronically published on November 4, 2008

\title{
A CONDITION FOR SPECTRAL CONTINUITY OF POSITIVE ELEMENTS
}

\author{
S. MOUTON
}

(Communicated by N. Tomczak-Jaegermann)

\begin{abstract}
Let $a$ be an element of a Banach algebra $A$. We introduce a compact subset $T(a)$ of the complex plane, show that the function which maps $a$ onto $T(a)$ is upper semicontinuous and use this fact to provide a condition on $a$ which ensures that if $\left(a_{n}\right)$ is a sequence of positive elements converging to $a$, then the sequence of the spectral radii of the terms $a_{n}$ converges to the spectral radius of $a$ in the case that $A$ is partially ordered by a closed and normal algebra cone and $a$ is a positive element.
\end{abstract}

\section{INTRODUCTION}

The subject of spectral continuity was essentially introduced by J. D. Newburgh in 15. Since then this fascinating topic has been studied widely, and several authors have made contributions: in particular by providing different types of sufficient conditions for spectral continuity, and by revealing the continuity of the restriction of the spectrum function or the spectral radius function to special subsets of certain Banach algebras. Most of the important earlier results were presented in [1], and in her survey paper [2] of 1994, L. Burlando gave an extensive account of these and subsequent results up to that time, supplying several useful references. Some of the more recent papers on the subject include [3], [5], 6] and [11].

A well-known example (see Section 4) illustrates, among other things, that if $A$ is an ordered Banach algebra, then the spectral radius function is in general not even continuous on its algebra cone. In (11], Corollary 4.9) it was shown, however, that if $a$ is a positive element, then the spectral radius function is continuous at $a$, considered as an element of the following subset of the algebra cone:

$$
\{y \in A: a \leq y, \text { and } a y \leq y a \text { or } y a \leq a y\} .
$$

A related problem which presents itself is that of discovering natural conditions on a positive element $a$ which will ensure that if $\left(a_{n}\right)$ is a sequence of positive elements converging to $a$, then the sequence of the spectral radii of the terms $a_{n}$ will converge to the spectral radius of $a$.

Let $A$ be a complex Banach algebra with unit 1 , and let $a \in A$. In this paper we define a compact subset $T(a)$ of the complex plane, which is related to the concept

Received by the editors June 29, 2007, and, in revised form, April 22, 2008, and July 21, 2008. 2000 Mathematics Subject Classification. Primary 46H05, 47A10.

Key words and phrases. Ordered Banach algebra, positive element, spectrum, upper semicontinuity.

The author thanks the referee for making useful suggestions.

(C)2008 American Mathematical Society Reverts to public domain 28 years from publication 
of the boundary spectrum of $a$ (i.e., the set of all complex numbers $\lambda$ such that $\lambda-a$ is contained in the topological boundary of the set of all non-invertible elements of $A$ ) as introduced in [12, and we establish some basic properties of $T(a)$. We then proceed to show that the map $a \mapsto T(a)$ is, in fact, upper semicontinuous. (Recall that the spectrum function itself is upper semicontinuous on $A$.) This fact is employed to obtain a condition as mentioned above in the case that $A$ is an ordered Banach algebra with a closed and normal algebra cone and $a$ is a positive element. We conclude by providing some examples.

\section{Preliminaries}

Throughout, $A$ will be a non-zero complex Banach algebra with unit 1 . The spectrum of an element $a$ in $A$ will be denoted by $\sigma(a)$ and the spectral radius of $a$ in $A$ by $r(a)$. If $\lambda \in \mathbb{C}$, the element $\lambda 1$ of $A$ will be denoted by $\lambda$.

Let $\mathbb{R}^{+}$denote the set of all non-negative real numbers and $C(z, r)$ the circle in the complex plane with centre $z$ and radius $r$. Also, for an $r>0$, an element $x$ and a set $E$ in a normed space $\mathcal{X}$, let $\mathcal{C} E$ denote the complement of $E$ in $\mathcal{X}$, $d(x, E)$ the distance from $x$ to $E, B(x, r)$ and $\bar{B}(x, r)$ the open and closed balls $\{y \in \mathcal{X}:\|x-y\|<r\}$ and $\{y \in \mathcal{X}:\|x-y\| \leq r\}$, respectively, and $\partial E$ the topological boundary of $E$.

Let $K(\mathbb{C})$ denote the set of compact subsets of the complex plane $\mathbb{C}$. We recall from ( 1 , pp. 48, 50) that the Hausdorff distance between two non-empty compact sets $K_{1}$ and $K_{2}$ in the complex plane is defined by

$$
\Delta\left(K_{1}, K_{2}\right)=\max \left\{\sup _{\lambda \in K_{1}} d\left(\lambda, K_{2}\right), \sup _{\lambda \in K_{2}} d\left(\lambda, K_{1}\right)\right\}
$$

and that a function $F: \mathcal{X} \rightarrow K(\mathbb{C}) \backslash \emptyset$ (with $\mathcal{X}$ a normed space) is upper semicontinuous on $\mathcal{X}$ if for every $x \in \mathcal{X}$ and every open set $U$ containing $F(x)$ there exists a $\delta>0$ such that if $\|y-x\|<\delta$, then $U$ contains $F(y)$. If we define $\Delta^{\prime}: K(\mathbb{C}) \times K(\mathbb{C}) \rightarrow \mathbb{R}^{+}$by

$$
\Delta^{\prime}\left(K_{1}, K_{2}\right)= \begin{cases}\frac{\Delta\left(K_{1}, K_{2}\right)}{1+\Delta\left(K_{1}, K_{2}\right)} & \text { if } K_{1}, K_{2} \neq \emptyset, \\ 0 & \text { if } K_{1}=K_{2}=\emptyset, \\ 1 & \text { otherwise, }\end{cases}
$$

then $\Delta^{\prime}$ induces on $K(\mathbb{C})$ the same topology as $\Delta$ on $K(\mathbb{C}) \backslash\{\emptyset\}$.

\section{ORDEREd Banach Algebras}

From ([16], Section 3) we recall that an algebra cone $C$ of a complex Banach algebra $A$ with unit 1 is a subset of $A$ containing 1 which is closed under the following operations: addition, non-negative real scalar multiplication, and multiplication. If $A$ has an algebra cone $C$, then $A$, or more specifically $(A, C)$, is called an ordered Banach algebra (OBA). If, in addition, $C \cap-C=\{0\}$, then $C$ is called proper.

An algebra cone $C$ of $A$ induces an ordering " $\leq$ " on $A$ in the following way:

$$
a \leq b \text { if and only if } b-a \in C
$$

(where $a, b \in A$ ). This ordering is reflexive and transitive. Furthermore, $C$ is proper if and only if the ordering has the additional property of being antisymmetric. Considering the ordering that $C$ induces we find that $C=\{a \in A: a \geq 0\}$ and therefore we call the elements of $C$ positive. 
An algebra cone $C$ of $A$ is called closed if it is a closed subset of $A$. Furthermore, $C$ is said to be normal if there exists a constant $\alpha>0$ such that it follows from $0 \leq a \leq b$ in $A$ that $\|a\| \leq \alpha\|b\|$. It is well known that if $C$ is normal, then $C$ is proper.

In [16] and [13, and later in [8]-12, some spectral theory of positive elements in ordered Banach algebras was developed. In particular, we recall the following result:

Theorem 3.1 ([16, Theorem 4.1(1)]). Let $(A, C)$ be an $O B A$ with $C$ normal. If $a, b \in A$ such that $0 \leq a \leq b$, then $r(a) \leq r(b)$.

We refer to the above property by saying that the spectral radius in $(A, C)$ is monotone.

Finally, we need the following example, where $\mathcal{L}(X)$ denotes the Banach algebra of all bounded linear operators on a Banach space $X$.

Example 3.2. Let $L$ be a non-zero complex Banach lattice and let $C:=\{x \in L$ : $x=|x|\}$. If $K:=\{T \in \mathcal{L}(L): T C \subseteq C\}$, then $K$ is a closed, normal algebra cone of $\mathcal{L}(L)$. Therefore $(\mathcal{L}(L), K)$ is an OBA.

The non-trivial part of the above example follows from ([17, Lemma 3).

\section{Spectral Continuity}

A well-known example by S. Kakutani about the discontinuity of the spectrum function also shows that in an ordered Banach algebra, the spectrum and the spectral radius functions are not continuous on the algebra cone:

Example 4.1 ([1, p. 49]). Let $l^{2}(\mathbb{N})$ be the complex Banach lattice of all squaresummable sequences. Then $\mathcal{L}\left(l^{2}(\mathbb{N})\right)$ is an OBA with a closed, normal algebra cone, say $K$, and there exist a sequence $\left(T_{n}\right)$ in $K$ and an operator $T \in K$ such that $\sigma\left(T_{n}\right)=\{0\}$ for all $n \in \mathbb{N}$ and $T_{n} \rightarrow T$ as $n \rightarrow \infty$, but $\sigma(T) \neq\{0\}$.

(The fact that $\mathcal{L}\left(l^{2}(\mathbb{N})\right)$ is an OBA with a closed, normal algebra cone follows from Example 3.2.)

Let $A$ be a complex Banach algebra with unit $1, S$ the (closed) set of all noninvertible elements of $A$ and $a \in A$. Define, as in [12, the boundary spectrum $S_{\partial}(a)$ (not to be confused with the peripheral spectrum $\{\lambda \in \mathbb{C}: \lambda \in \sigma(a)$ and $|\lambda|=r(a)\}$ ) of $a$ as follows:

$$
S_{\partial}(a)=\{\lambda \in \mathbb{C}: \lambda-a \in \partial S\} .
$$

Then $S_{\partial}(a)$ is compact and non-empty, $S_{\partial}(a) \subseteq \bar{B}(0, r(a))$ and $\partial \sigma(a) \subseteq S_{\partial}(a) \subseteq$ $\sigma(a)$ ([12, Proposition 2.1). In general, $\partial \sigma(a) \neq S_{\partial}(a)$ (12], Example 2.3). Several additional properties of the boundary spectrum were established in [12. In particular, we recall the following result:

Proposition 4.2 ([12, Proposition 3.3]). Let $(A, C)$ be an $O B A$ with $C$ closed and such that the spectral radius in $(A, C)$ is monotone. If $a \in C$, then $r(a) \in S_{\partial}(a)$.

We now define another set, $T(a)$, in the complex plane, as follows:

$$
T(a)=\{\lambda \in \mathbb{C}:|\lambda|-a \in \partial S\} .
$$

Clearly, $\lambda \in T(a)$ if and only if $|\lambda| \in T(a)$. Furthermore,

$$
T(a)=\left\{\lambda \in \mathbb{C}:|\lambda| \in S_{\partial}(a)\right\}=\left\{\lambda \in \mathbb{C}:|\lambda| \in S_{\partial}(a) \cap \mathbb{R}^{+}\right\} .
$$


Hence $T(a)$ is a compact set and $T(a) \subseteq \bar{B}(0, r(a))$. Also, if $\lambda_{0} \in \mathbb{R}^{+}$, then $S_{\partial}(a) \cap \mathbb{R}^{+}=\left\{\lambda_{0}\right\}$ if and only if $T(a)=C\left(0, \lambda_{0}\right)$. If $T(a) \neq \emptyset$ and $\gamma(a)=\sup \{|\lambda|$ : $\lambda \in T(a)\}$, then $\gamma(a) \in T(a)$ for all $a \in A$. We have the following lemma:

Lemma 4.3. Let $(A, C)$ be an $O B A$ with $C$ closed and normal. If $a \in C$, then $\gamma(a)=r(a)$ and $r(a) \in T(a)$.

Proof. Since $T(a) \subseteq \bar{B}(0, r(a))$ for all $a \in A$, it is always the case (if $T(a) \neq \emptyset$ ) that $\gamma(a) \leq r(a)$. If $a \in C$, then by Theorem 3.1 and Proposition 4.2, $r(a) \in S_{\partial}(a)$. Since $r(a) \in \mathbb{R}^{+}$, it follows that $r(a) \in T(a)$, so that $T(a) \neq \emptyset$ and $r(a) \leq \gamma(a)$.

It follows that for a positive element $a \in A$, the set $T(a)$ is not empty. The following example illustrates that the situation $T(a)=\emptyset$ can occur for non-positive elements, and that if $a \notin C$, then $\gamma(a)=r(a)$ and $r(a) \in T(a)$ may not hold.

Example 4.4. Let $A$ be the set of upper triangular $2 \times 2$ complex matrices and $C$ the subset of $A$ of matrices with only non-negative entries. Then $(A, C)$ is an OBA and $C$ is closed and normal. Also, there exist

(1) an $a_{1} \notin C$ such that $T\left(a_{1}\right)=\emptyset$, and

(2) an $a_{2} \notin C$ such that $T\left(a_{2}\right) \neq \emptyset$, but $r\left(a_{2}\right) \notin T\left(a_{2}\right)$ and $\gamma\left(a_{2}\right) \neq r\left(a_{2}\right)$.

Proof. The first statement follows from (8], Example 4.8). In this case $\partial S=S$ and hence, if $a=\left(\begin{array}{cc}\lambda_{1} & \lambda_{2} \\ 0 & \lambda_{4}\end{array}\right) \in A$, then $S_{\partial}(a)=\left\{\lambda_{1}, \lambda_{4}\right\}=\sigma(a)$ and $T(a)=\{\lambda \in \mathbb{C}$ : $|\lambda|=\lambda_{1}$ or $\left.|\lambda|=\lambda_{4}\right\}$.

(1) Let $a_{1}=\left(\begin{array}{rr}-1 & 1 \\ 0 & -1\end{array}\right)$. Then $a_{1} \notin C$ and $T\left(a_{1}\right)=\emptyset$.

(2) Let $a_{2}=\left(\begin{array}{rr}-3 & 1 \\ 0 & 1\end{array}\right)$. Then $a_{2} \notin C, r\left(a_{2}\right)=3$ and $T\left(a_{2}\right)=\{\lambda \in \mathbb{C}:|\lambda|=1\}$, so that $T\left(a_{2}\right) \neq \emptyset$ and $\gamma\left(a_{2}\right)=1$. It follows that $r\left(a_{2}\right) \notin T\left(a_{2}\right)$ and $\gamma\left(a_{2}\right) \neq r\left(a_{2}\right)$. (We note, however, that $\gamma\left(a_{2}\right) \in T\left(a_{2}\right)$, as mentioned before.)

In fact, if $A$ is any complex Banach algebra with unit 1 , then $T(-1)=\{\lambda \in \mathbb{C}$ : $|\lambda| \in\{-1\}\}=\emptyset$. It follows that if $(A, C)$ is any OBA with $C$ closed and normal, then there exists an $a \notin C$ such that $T(a)=\emptyset$.

The following theorem states a particularly useful property of the map $a \mapsto T(a)$.

Theorem 4.5. Let $A$ be a Banach algebra. The function $a \mapsto T(a)$ from $A$ into $K(\mathbb{C})$ is upper semicontinuous on $A$.

Proof. If not, then there exist an $a \in A$, an open set $U$ containing $T(a)$ and for each $n \in \mathbb{N}$ an $a_{n} \in A$ such that $a_{n} \rightarrow a$ as $n \rightarrow \infty$, but $T\left(a_{n}\right) \nsubseteq \mathbb{U}$, say $\lambda_{n} \in T\left(a_{n}\right) \cap \mathbb{C} \backslash U$.

From $\lambda_{n} \in T\left(a_{n}\right)$ it follows that $\left|\lambda_{n}\right| \leq r\left(a_{n}\right) \leq\left\|a_{n}\right\| \leq K$ (say), for all $n \in \mathbb{N}$, since $\left(a_{n}\right)$ is a convergent sequence. By the Bolzano-Weierstrass Theorem, $\left(\lambda_{n}\right)$ has a convergent subsequence, say $\lambda_{n_{k}} \rightarrow \lambda$ as $k \rightarrow \infty$.

Since $\lambda_{n} \notin U$ and $U$ is open, it follows that $\lambda \notin U$. Hence $\lambda \notin T(a)$, so that $|\lambda|-a \notin \partial S$. It follows that for some $\epsilon>0$,

$$
\text { either } B(|\lambda|-a, \epsilon) \subseteq S \text { or } B(|\lambda|-a, \epsilon) \subseteq \mathcal{C} S \text {. }
$$

Since $\lambda_{n_{k}} \rightarrow \lambda$ and $a_{n_{k}} \rightarrow a$ as $k \rightarrow \infty$, there is an $N \in \mathbb{N}$ such that $\left|\lambda_{N}\right|-$ $a_{N} \in B(|\lambda|-a, \epsilon)$. Let $\rho=\epsilon-\left\|\left(\left|\lambda_{N}\right|-a_{N}\right)-(|\lambda|-a)\right\|$. Then $\rho>0$ and $B\left(\left|\lambda_{N}\right|-a_{N}, \rho\right) \subseteq B(|\lambda|-a, \epsilon)$. 
Since $\lambda_{N} \in T\left(a_{N}\right)$, we have that $\left|\lambda_{N}\right|-a_{N} \in \partial S$, so that $B\left(\left|\lambda_{N}\right|-a_{N}, \rho\right)$ contains a point of $S$ as well as a point of $\mathcal{C} S$. But then $B(|\lambda|-a, \epsilon)$ contains points of both $S$ and $\mathcal{C} S$, which contradicts (4.1). Hence the result follows.

Using the upper semicontinuity of the map $a \mapsto T(a)$, we can now establish our main result.

Theorem 4.6. Let $(A, C)$ be an $O B A$ with $C$ closed and normal, and let $a \in C$ be such that $S_{\partial}(a) \cap \mathbb{R}^{+}=\{r(a)\}$ (or, equivalently, $T(a)=C(0, r(a))$ ). If $\left(a_{n}\right)$ is a sequence in $C$ such that $a_{n} \rightarrow a$ as $n \rightarrow \infty$, then $r\left(a_{n}\right) \rightarrow r(a)$ as $n \rightarrow \infty$.

Proof. Let $\left(\lambda_{n}\right)$ be a sequence in $T(a)$ such that $d\left(r\left(a_{n}\right), T(a)\right)=\left|r\left(a_{n}\right)-\lambda_{n}\right|$.

Let $\epsilon>0$ and $U=\{\lambda \in \mathbb{C}: d(\lambda, T(a))<\epsilon\}$. Then $U$ is open and $T(a) \subseteq U$. Since $a_{n} \rightarrow a$ and the map $x \mapsto T(x)$ is upper semicontinuous at $a$, there exists an $N \in \mathbb{N}$ such that if $n \geq N$, then $T\left(a_{n}\right) \subseteq U$. By Lemma 4.3, $r\left(a_{n}\right) \in T\left(a_{n}\right)$ for all $n \in \mathbb{N}$, and therefore $\left|r\left(a_{n}\right)-\lambda_{n}\right|<\epsilon$ for all $n \geq N$. It follows that $\left|r\left(a_{n}\right)-\right| \lambda_{n}||<\epsilon$ for all $n \geq N$. Since $\lambda_{n} \in T(a)$, it follows that $\left|\lambda_{n}\right| \in S_{\partial}(a) \cap \mathbb{R}^{+}$, so that $\left|\lambda_{n}\right|=r(a)$, for all $n \in \mathbb{N}$. Therefore $\left|r\left(a_{n}\right)-r(a)\right|<\epsilon$ for all $n \geq N$.

(In the above proof we could have assumed, in fact, that $\lambda_{n}=r(a)$ for all $n \in \mathbb{N}$, since $T(a)=C(0, r(a))$.)

Finally, we consider some examples.

Example 4.7 ([7, Problem 84]). Let $l^{2}(\mathbb{Z})$ be the complex Banach lattice of all bilateral square-summable sequences and $W: l^{2}(\mathbb{Z}) \rightarrow l^{2}(\mathbb{Z})$ the bilateral shift

$$
W\left(\ldots, \xi_{-2}, \xi_{-1},\left(\xi_{0}\right), \xi_{1}, \xi_{2}, \ldots\right)=\left(\ldots, \xi_{-3}, \xi_{-2},\left(\xi_{-1}\right), \xi_{0}, \xi_{1}, \xi_{2}, \ldots\right),
$$

where the term in round brackets indicates the one corresponding to index zero. Then $\sigma(W)=C(0,1)$.

By Example 3.2, $\mathcal{L}\left(l^{2}(\mathbb{Z})\right)$ is an OBA with a closed, normal algebra cone, say $K$. Clearly $W \in K$. For $W$ we have $S_{\partial}(W)=\sigma(W)=C(0,1)$, so that $S_{\partial}(W) \cap \mathbb{R}^{+}$ consists of the spectral radius $r(W)=1$ only. Hence Theorem 4.6 is applicable in this case. Here, however, the continuity of the spectral radius function at $W$ follows already from (11), Proposition 4.11), since $\sigma(W) \subseteq C(0, r(W)$ ). (We note that (11], Proposition 4.11) is, in fact, an immediate corollary of ([14, Proposition 1).)

The following lemma will provide additional examples of positive elements where Theorem 4.6 can be used.

Lemma 4.8. Let $(A, C)$ be an $O B A$ with $C$ closed and normal, and let $a \in C$ be such that $\sigma(a)=C(0,1)$. If $\lambda>0$ and $b_{\lambda}=a+\lambda$, then $b_{\lambda} \in C$ and $b_{\lambda}$ has the following properties:

(1) $\sigma\left(b_{\lambda}\right)=C(\lambda, 1)$ and hence $r\left(b_{\lambda}\right)=\lambda+1$.

(2) $\sigma\left(b_{\lambda}\right) \nsubseteq \subseteq\left(0, r\left(b_{\lambda}\right)\right)$ and if, in addition, $\lambda<1$, then $S_{\partial}\left(b_{\lambda}\right) \cap \mathbb{R}^{+}=\left\{r\left(b_{\lambda}\right)\right\}$.

Proof. (1) This follows from the Spectral Mapping Theorem.

(2) If $\mu=\lambda-1$, then $\mu \in \sigma\left(b_{\lambda}\right) \backslash C\left(0, r\left(b_{\lambda}\right)\right)$. Since $\sigma\left(b_{\lambda}\right)=C(\lambda, 1)$, we have that $\partial \sigma\left(b_{\lambda}\right)=\sigma\left(b_{\lambda}\right)$, so that $S_{\partial}\left(b_{\lambda}\right)=\sigma\left(b_{\lambda}\right)=C(\lambda, 1)$. If $\lambda<1$, then $\lambda-1<0$, so that $S_{\partial}\left(b_{\lambda}\right) \cap \mathbb{R}^{+}=C(\lambda, 1) \cap \mathbb{R}^{+}=\{\lambda+1\}=\left\{r\left(b_{\lambda}\right)\right\}$.

For an element $a$ in any Banach algebra $A$, let

$$
\alpha(a)=\sup \{\inf \{|\mu|: \mu \in \omega\}: \omega \text { is a component of } \sigma(a)\}
$$


(see [14, p. 620). Then the equivalence of (i) and (ii) in ([14, Proposition 1) implies that $\alpha(a)=r(a)$ is a sufficient condition for the spectral radius $r$ to be continuous at $a$.

On the other hand, if $(A, C)$ is an OBA with $C$ closed and normal and $a \in C$, then Theorem 4.6] states that $S_{\partial}(a) \cap \mathbb{R}^{+}=\{r(a)\}$ is a sufficient condition for the restriction of $r$ to $C$ to be continuous at $a$.

Now consider any OBA $(A, C)$ with $C$ closed and normal. If $a \in C$ is such that $\sigma(a)=C(0,1)$ and $b_{\lambda}=a+\lambda$ with $0<\lambda<1$, then $\alpha\left(b_{\lambda}\right)=1-\lambda<1+\lambda=$ $r\left(b_{\lambda}\right)$. Hence $b_{\lambda}$ does not satisfy the sufficient condition for continuity of $r$ at $b_{\lambda}$ given in ([14], Proposition 1). According to Lemma 4.8, $b_{\lambda}$ does, however, satisfy the sufficient condition for continuity of the restriction of $r$ to $C$ at $b_{\lambda}$ given in Theorem 4.6 .

If, in addition, $(A, C)=\left(\mathcal{L}\left(l^{2}(\mathbb{Z})\right), K\right)$ (see Example 4.7), then it follows from (4], Theorem 2.6) that $r$ is not continuous at $b_{\lambda}$. However, Lemma 4.8 and Theorem 4.6 imply that the restriction of $r$ to $C$ is continuous at $b_{\lambda}$.

\section{REFERENCES}

1. B. Aupetit, A Primer on Spectral Theory, Springer, New York, 1991. MR.1083349(92c:46001)

2. L. Burlando, Continuity of spectrum and spectral radius in Banach algebras, in: Functional Analysis and Operator Theory, J. Zemánek (ed.), Banach Center Publ. 30, Inst. Math., Polish Acad. Sci., Warsaw, 1994, 53-100. MR 1285600 (95i:46062)

3. L. Burlando, Noncontinuity of spectrum for the adjoint of an operator, Proc. Amer. Math. Soc. 128 (2000), 173-182. MR1625705 (2000c:47007)

4. J. B. Conway and B. B. Morrel, Operators that are points of spectral continuity, Integral Equations Operator Theory 2 (1979), 174-198. MR543882 (80h:47004)

5. S. V. Djordjević and Y. M. Han, Browder's theorems and spectral continuity, Glasg. Math. J. 42 (2000), 479-486. MR1793814 (2001h:47003)

6. S. V. Djordjević and Y. M. Han, Spectral continuity for operator matrices, Glasg. Math. J. 43 (2001), 487-490. MR1878591 (2002k:47008)

7. P. R. Halmos, A Hilbert Space Problem Book, Graduate Texts in Math., vol. 19, Springer, New York, 1982. MR675952(84e:47001)

8. H. du T. Mouton and S. Mouton, Domination properties in ordered Banach algebras, Studia Math. 149 (2002), 63-73. MR1881716 (2003e:46076)

9. S. Mouton, A spectral problem in ordered Banach algebras, Bull. Austral. Math. Soc. 67 (2003), 131-144. MR.1962967 (2004d:47075)

10. S. Mouton, Convergence properties of positive elements in Banach algebras, Math. Proc. R. Ir. Acad. Sect. A 102 (2002), 149-162. MR.1961634(2004d:47074)

11. S. Mouton, On spectral continuity of positive elements, Studia Math. 174 (2006), 75-84. MR2239814 (2007c:46047)

12. S. Mouton, On the boundary spectrum in Banach algebras, Bull. Austral. Math. Soc. 74 (2006), 239-246. MR2260492(2007g:46072)

13. S. Mouton (née Rode) and H. Raubenheimer, More spectral theory in ordered Banach algebras, Positivity 1 (1997), 305-317. MR1660397 (2000a:46070)

14. G. J. Murphy, Continuity of the spectrum and spectral radius, Proc. Amer. Math. Soc. 82 (1981), 619-621. MR614889 (82h:46066)

15. J.D. Newburgh, The variation of spectra, Duke Math. J. 18 (1951), 165-176. MR0051441 $(14: 481 \mathrm{~b})$

16. H. Raubenheimer and S. Rode, Cones in Banach algebras, Indag. Math. (N.S.) 7 (1996), 489-502. MR1620116 (99i:46035)

17. H. H. Schaefer, Some spectral properties of positive linear operators, Pacific J. Math. 10 (1960), 1009-1019. MR0115090 (22:5893)

Department of Mathematical Sciences, University of Stellenbosch, Private Bag X1, Matieland 7602, South Africa

E-mail address: smo@sun.ac.za 